University of Nebraska - Lincoln

DigitalCommons@University of Nebraska - Lincoln

8-2002

\title{
Use of Exemplar Surveys to Reveal Implicit Types of Intelligence
}

Delroy L. Paulhus

University of British Columbia Faculty of Medicine, dpaulhus@psych.ubc.ca

Paul Wehr

University of British Columbia

Peter D. Harms

University of Nebraska - Lincoln, pharms@gmail.com

David I. Strasser

University of British Columbia

Follow this and additional works at: https://digitalcommons.unl.edu/leadershipfacpub

Part of the Management Sciences and Quantitative Methods Commons

Paulhus, Delroy L.; Wehr, Paul; Harms, Peter D.; and Strasser, David I., "Use of Exemplar Surveys to Reveal Implicit Types of Intelligence" (2002). Leadership Institute Faculty Publications. 15.

https://digitalcommons.unl.edu/leadershipfacpub/15

This Article is brought to you for free and open access by the Leadership Institute at DigitalCommons@University of Nebraska - Lincoln. It has been accepted for inclusion in Leadership Institute Faculty Publications by an authorized administrator of DigitalCommons@University of Nebraska - Lincoln. 
Published in Personality and Social Psychology Bulletin 28:8 (August 2002), pp. 1051-1062; doi: 10.1177/01461672022811004 Copyright @ 2002 Society for Personality and Social Psychology, Inc.; published by Sage Publications. Used by permission. http://psp.sagepub.com

\title{
Use of Exemplar Surveys to Reveal Implicit Types of Intelligence
}

\author{
Delroy L. Paulhus \\ Paul Wehr \\ P. D. Harms \\ David I. Strasser \\ University of British Columbia
}

\begin{abstract}
Implicit theories of intelligence were investigated via surveys of exemplars of intelligence. Study 1 was a four-sample survey of famous exemplars. These diverse samples reported a similar set of popular exemplars, which clustered into five groups. These groups represented five types of intelligence: scientific, artistic, entrepreneurial, communicative, and moral intelligence. In Study 2, the minimal overlap of intelligence exemplars with those of fame, creativity, and wisdom refuted the possibility that exemplar reports are indiscriminate or solely a result of availability. In Study 3, knowledgeable judges rated the similarity of 50 famous persons to exemplars representing each type of intelligence. All five similarity ratings predicted exemplar popularities. In Study 4, where exemplar reports were not restricted to famous people, $31 \%$ were nonfamous (friends, family members, teachers, etc.). The results indicate that five implicit types of intelligence, each represented by highly available exemplars, play a role in people's implicit theories.
\end{abstract}

Research on implicit theories of intelligence addresses lay perceptions and conceptions of intelligence. The topic is typically contrasted with the more traditional research on explicit theories of intelligence, that is, claims about the nature of cognitive performance and individual differences therein. It can be argued that research on implicit theories is actually the more important approach because its influence extends to everyday social interactions, voting preferences, and coping as well as scholastic and job evaluations (Hogan \& Hogan, 1994; Hong, Chiu, \& Dweck, 1995; Sternberg, 1988).
Interest in the topic began with two 1979 articles that proposed the cognitive representation of intelligence as a prototype (Cantor \& Mischel, 1979; Neisser, 1979). The prototype model suggests that we store a template of attributes representing our ideal of an intelligent person and we judge others as intelligent to the extent that they match the attributes in our prototype of intelligence (Neisser, 1979). Extensive work by Sternberg and his colleagues revealed substantial empirical support for these propositions (Sternberg, 1985; Sternberg, Conway, Ketron, \& Bernstein, 1981). Their methods were largely straightforward: Lay judges were asked what they mean by the term intelligent and other mental abilities. Among other things, this research demonstrated that prototypes of intelligence are distinct from those of related mental abilities such as creativity and wisdom. ${ }^{1}$ This early prototype work has since been followed up by others studying implicit theories of intelligence (e.g., Kosmitzki \& John, 1993; Raty \& Snellman, 1997; Ruisel, 1996) and personality traits (Broughton, 1984; Chaplin, John, \& Goldberg, 1988; John, 1986; Rush \& Russell, 1988).

An alternative to prototype theory is exemplar theory (e.g., Nosofsky, 1992; Smith \& Zarate, 1992). Here, an individual's cognitive representation of the trait "intelligent" is assumed to include memories of intelligent individuals with whom the perceiver has had experience. As far as we know, the only empirical work on intelligence from an exemplar perspective comes from our own laboratory (Paulhus, 2000; Paulhus \& Landolt, 2000). There we examined the stability across 16 years

The authors would like to thank Nadine Bruce, Monica Landolt, David Lim, Kathy Morgan, Gordon Murphy, and Doug Reid for assistance in collecting and compiling the data. Sam Gosling provided valuable comments on a previous draft. This research was supported by a grant from the Social Science and Humanities Research Council of Canada.

Corresponding author - Del Paulhus, Department of Psychology, University of British Columbia, 2136 West Mall, Vancouver, Canada V6T 1Z4; email $\underline{\text { dpaulhus@psych.ubc.ca }}$ 
of famous exemplars reported by college students. The rationale was that the popularity of exemplars reveals something about a culture's conception of intelligence. Such popular exemplars included Albert Einstein, U.S. President, Da Vinci, and Shakespeare. The top 15 accounted for $83 \%$ of the exemplar reports. Those data suggest that a small set of shared exemplars play a role in our conceptions of intelligence.

In the present report, we pursued the promise of an exemplar approach in four studies. In particular, we collected several types of nomination and rating data to evaluate the generalizability and discriminant validity of the Paulhus and Landolt (2000) findings. In Study 1, participants were simply asked to name a famous example of an intelligent person. The results were tabulated and compared across a variety of nominator samples. Study 2 examined whether judges could discriminate among intelligent, creative, and wise exemplars. Study 3 investigated various attributes of candidates likely to influence their probability of being nominated. In Study 4, exemplar reports were left unrestricted to explore the influence of nonfamous exemplars.

\section{Study 1: How Consensual Are Famous Exemplars?}

The temporal consistency in famous exemplars found by Paulhus and Landolt (2000) might be attributable to sampling students in the same school. To evaluate consistency across more diverse samples, we compiled exemplar reports from four sources: (a) 244 students at the University of California, Berkeley; (b) 153 students at the University of Georgia; (c) 401 students at the University of British Columbia; and (d) 351 adults from the Vancouver, Canada, area. Participants were asked to give an ideal example of a famous intelligent person. Results were compiled and used to facilitate subsequent classroom discussion of the concept of intelligence.

\section{Method}

The instructor solicited exemplar reports from the student samples before the topic of intelligence was covered in lectures. The topic was not in any of the textbooks and, therefore, did not contaminate the exemplar reports. The exemplar reports were voluntary, although less than $1 \%$ declined. Responses were submitted on a sheet of paper along with the nominator's sex and year in university.

Student data $(N=798)$ were collected from undergraduate psychology classes. The sex breakdown was similar across samples, ranging from $60 \%$ to $66 \%$ female. Sex differences in exemplar reports were neither clear nor consistent; therefore, the data were combined across sex (with the exceptions specifically noted).

Data collection began with the following instructions being read aloud:
Think of an ideal example of an intelligent person. Not a friend or family member, but someone who is well known-alive or not. Write the name on a sheet of paper and hand it in. Don't put your name or any identification on it. I will compile the names and give you the results of the survey in the next class.

The adult data $(N=351)$ were collected by asking students to solicit an exemplar report from one of their parents or some other individual older than age 30 . The student took home a form with instructions similar to the above. The form asked the adult nominators to indicate their relationship with the student and their phone number. The latter requirement has been found in the past to ensure that the reports were actually completed by the adults (Paulhus, Lysy, \& Yik, 1998). In the present adult sample, 55\% were women and 70\% were family members.

\section{Results and Discussion}

Exemplar report frequencies. Table 1 provides the top 15 nominees for the four samples: (a) a 1980s student sample from a Southeastern U.S. university, (b) a 1990s sample from a West Coast U.S. university, (c) a 1990s sample from a Northwestern Canadian university, and (d) a 1980s adult sample from Vancouver, Canada. The similarity across samples is striking, particularly at the top of the lists. Eight individuals appear on all four: Einstein, U.S. President, Da Vinci, Isaac Newton, Shakespeare, Mozart, Oprah Winfrey, and Stephen Hawking. Two others, the contemporary Canadian and British Prime Ministers, appeared in the top 15 for the two Canadian samples. The similarity across samples was indexed by taking the top 20 from each list (36 in total) and correlating their frequencies across the four samples. All intercorrelations exceeded $r=.81, p<.01$.

The student lists were particularly close: Across time, across countries, and across schools, the most popular exemplars were consistent. The adult list is also similar but reflects cultural and generational differences: It included three Americans (Walter Cronkite, Bob Dylan, and Henry Kissinger) and one Canadian (Pierre Berton) who do not appear on the student lists - presumably because their influence has diminished considerably over the last generation. When the lists were restricted to living individuals, another interesting pattern emerged. Here, the U.S. president led all four lists. In the Canadian samples, the Canadian Prime Minister ranked second. Clearly, national leaders are among the most prominent exemplars of intelligence.

Clusters of famous exemplars. Despite the consistency across samples, the diversity within popular nominees is striking. Consider how the very concept of intelligence appears to shift across such popular nominees as Einstein, the U.S. President, Shakespeare, Bill Gates, Mo- 
Table 1. The Top 15 Exemplars of Intelligence (with percentage of total reports) in Study 1

\begin{tabular}{llll}
\hline U. of California & U. of Georgia & Canadian Students & Canadian Adults \\
Students (N = 244) & Students $(\mathrm{N}=153)$ & $(\mathrm{N}=401)$ & $351)$ \\
Fall 1996 & Fall 1981 & $1982-1997$ & 1988, 1992 \\
\hline Einstein (30) & Einstein (33) & Einstein (27) & Einstein (25) \\
U.S. President (11) & U.S. President (10) & U.S. President (8) & C.S. President (9) \\
Thomas Edison (7) & Da Vinci (6) & Canadian P.M. (7) & Canadian P.M. (6) \\
Stephen Hawking (6) & Shakespeare (5) & Da Vinci (5) & Shakespeare (6) \\
Bill Gates (6) & Thomas Edison (5) & Isaac Newton (5) Edison (3) \\
Isaac Newton (4) & Isaac Newton (5) & Bill Gates (5) & Da Vinci (3) \\
Da Vinci(4) & Oprah Winfrey (4) & Stephen Hawking (5) & Henry Kissinger (3) \\
Wolfgang Mozart (3) & Lee Iacocca (4) & Shakespeare (4) & Isaac Newton (3) \\
Shakespeare (3) & British P.M. (3) & Thomas Edison (4) & Wolfgang Mozart (2) \\
Oprah Winfrey (3) & Donald Trump (3) & Oprah Winfrey (4) & Pierre Berton (2) \\
Sigmund Freud (3) & Wolfgang Mozart (3) & British P.M. (3) & Walter Cronkite (2) \\
Stephen Spielberg (2) & Sigmund Freud (3) & Wolfgang Mozart (2) & Stephen Hawking (2) \\
Diane Feinstein (2) & Carl Sagan (2) & David Suzuki (2) & Oprah Winfrey (2) \\
Malcolm X (2) & M. L. King (2) & Sigmund Freud (2) & Bob Dylan (2) \\
Noam Chomsky (2) & Jimmy Carter (2) & Madonna (2) & British P.M. (2)
\end{tabular}

P.M. = prime minister

zart, Oprah Winfrey, and Martin Luther King. To clarify this diversity, we conducted a multidimensional scaling analysis of the top 15 nominees. $^{2}$

A sample of 12 knowledgeable judges were asked to rate the pairwise similarity of the 15 exemplars with respect to their intelligence. The sample of judges was limited to faculty members and graduate students to ensure a reasonable familiarity with all of the exemplars. All confirmed that they were quite familiar with the 15 exemplars. The 10-point rating scale was anchored by the labels minimal (1) and maximal (10). The interrater intercorrelations across the 105 similarity ratings ranged from .59 to 1.00 , with a mean of .75 . As a panel, the raters yielded an alpha of .96, indicating sufficient similarity in the judges' perceptions to combine their ratings.

The mean of each of the 105 pairwise similarities was calculated across the 12 judges. These similarities were submitted to SPSS-ALSCAL to perform a multidimensional scaling of the 15 exemplars. The two-dimensional solution, presented in Figure 1, yielded interpretable clusters in the minimal number of dimensions with an acceptable stress value (.04). Dimension 1 (horizontal) can be loosely interpreted as public versus private communication running from those working privately (scientists, writers) to those working publicly (politicians, etc.). Dimension 2 (vertical) is loosely interpretable as a subjective versus physical domain, that is, a qualitative shift from those dealing with the subjective (Mozart, Gandhi) to those dealing with the physical world (Edison, Gates, Ted Turner).

More illuminating than the dimensions were the clusters themselves. Figure 1 reveals that the exemplars have separated into five distinctive clusters: We labeled them scientific intelligence (Einstein, Hawking), artistic

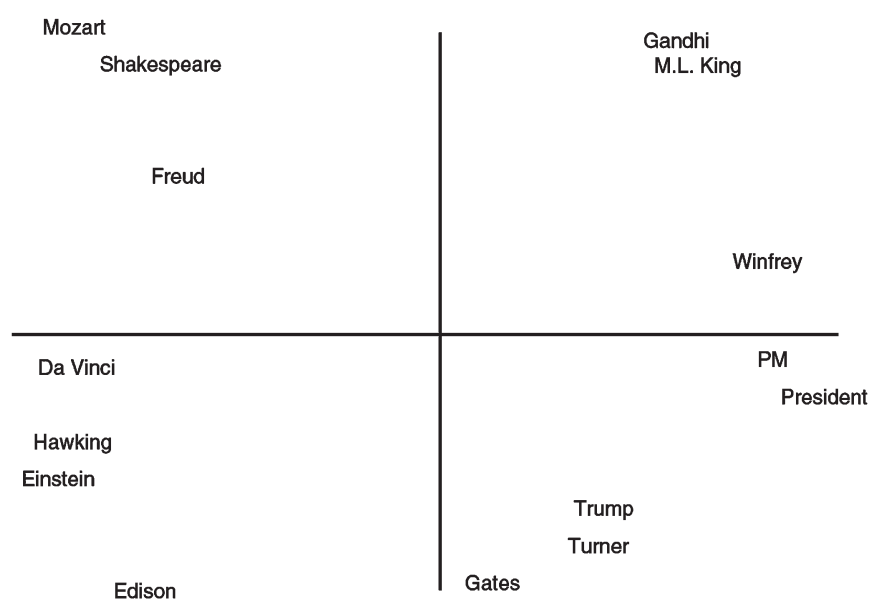

Figure 1. Two-dimensional solution from a multidimensional scaling of 15 exemplars. (PM = prime minister)

intelligence (Mozart, Shakespeare), entrepreneurial intelligence (Turner, Trump, Gates), communicative intelligence (President, Prime Minister, Winfrey), and moral intelligence (e.g., Gandhi, M. L. King).

\section{Conclusion}

There was a marked similarity across samples in the most popular exemplars of intelligence. Only minor differences appeared across various educated samples in North America. Within these popular exemplars, a diversity of worldviews was reflected in the emergence of five clusters. Consistent with earlier work (Gardner, 1998; Sternberg, 1988), intelligence was found to be a heterogeneous concept even within educated samples. Unlike earlier work, we isolated five types of intelli- 
Table 2. Nominations for Intelligent, Creative, Wise, and Famous Persons in Study 2

\begin{tabular}{|c|c|c|c|c|}
\hline & Intelligent & Creative & Wise & Sheer Fame \\
\hline 2. & Bill Clinton & Picasso & Confucius & Elvis Presley \\
\hline 4. & Prime Minister & Mozart & M. L. King & Muhammad Ali \\
\hline 5. & Gates & Spielberg & Socrates & Michael Jackson \\
\hline 6. & Shakespeare & Shakespeare & Mother Theresa & Bill Clinton \\
\hline 9. & Newton & Walt Disney & Pope & Bill Gates \\
\hline 10. & Mozart & Robin Williams & Oprah Winfrey & John F. Kennedy \\
\hline 11. & Edison & Salvador Dali & Winston Churchill & Nelson Mandela \\
\hline 12. & Suzuki & Madonna & Dali Lama & Marilyn Monroe \\
\hline 13. & Madonna & Sigmund Freud & Ann Landers & Adolph Hitler \\
\hline 14. & Gorbachev & Alexander Graham Bell & Nelson Mandela & George Bush, Sr. \\
\hline
\end{tabular}

Total $N=486$.

gence considered to be important and distinctive by our lay and expert judges.

The ultimate value of such exemplar analyses lies in showing that people can make use of the distinctions emerging here: This utility is evaluated in Studies 3 and 4. First, the issue of discriminant validity warrants some attention.

\section{Study 2: Discriminant Validity - Intelligence Versus Creativity, Wisdom, and Sheer Fame}

Intelligence, along with creativity, and wisdom may be subsumed into a higher level category, namely, mental ability (Sternberg, 1985). Data collected within the prototype paradigm showed that expert and lay judges alike made clear distinctions among the concepts of intelligence, creativity, and wisdom (Holliday \& Chandler, 1986). Presumably, it has proven useful to retain such distinctions in the English language as well as in the shared cognition of Western culture (Ruzgis \& Grigorenko, 1994; Thompson \& Fine, 1999). If ideal exemplars are linked to or are parallel to these three prototypes, then similar distinctions should appear among exemplars of intelligent, creative, and wise persons.

It is particularly important to establish this distinctiveness given the present self-report method of identifying exemplars. The sheer salience of certain individuals in our society may fully explain why they dominated the exemplar reports (Tversky \& Kahneman, 1974). The most available names will naturally be the most nominated. Perceptions of fame are sensitive even to rather minor influences (e.g., Banaji \& Greenwald, 1995; Jacoby, Kelley, Brown, \& Jasechko, 1989). To directly address the potential fame confound, we also solicited exemplars of famous persons without reference to their intelligence: They should personify sheer fame.

\section{Method and Results}

Several large undergraduate classes were surveyed in 1993. The procedure was similar to that described in Study 1, with two exceptions. First, the type of exemplar requested was manipulated across three conditions ( $n$ $=101$ per cell). Participants were randomly assigned to conditions by numbering off $(1,2,3,1,2,3 \ldots)$ along each row in the class. Those in Condition 1 were asked to provide an exemplar of intelligence, in Condition 2 an exemplar for creativity, and in Condition 3 an exemplar for wisdom. For comparison, a sheer fame condition ( $n$ $=183$ ) was created from students in a separate section of the same course: They were asked simply to nominate a famous person.

The top 15 from each condition are listed in Table 2. Four individuals from the creativity list overlapped with the intelligence list: Da Vinci, Mozart, Shakespeare, and Madonna. Only one individual on the wise list (Oprah Winfrey) also appeared on the intelligence list. ${ }^{3}$ No individual appeared on both the creativity and wisdom lists.

This pattern of overlap is entirely consistent with Sternberg's work on prototypical features. Similar to Sternberg, we found that overlap with the intelligent exemplars was higher for those on the creative list $(27 \%)$ than for those on the wisdom list $(7 \%)$. And the zero overlap of the top 15 exemplars for creativity and wisdom matches Sternberg's (1988) demonstration of minimal conceptual overlap between creativity and wisdom.

Overall, there is no indication from these results that our student judges are confusing intelligence with related mental abilities. This discriminability is sufficient to refute suspicions that sheer fame accounts for high nomination rates as exemplars of intelligence. Had this been the case, the same individuals would have ap- 
peared on the intelligence, creativity, and wisdom lists. Note that we chose a between-subjects design to avoid the possibility that nominators might bypass their best exemplar for intelligence simply to save him/her for nomination as a creative or wise person.

Finally, the list comprising exemplars of pure fame overlapped no more than $20 \%$ with any of the three mental ability lists. Instead, the individuals heading the fame list (Princess Diana, Elvis Presley, and Michael Jordan) appeared on none of the ability lists.

Together, these results make it clear that famous exemplars of intelligence are more than just famous. Their exemplar frequencies are determined by the specific nature of their (perceived) mental abilities. Study 3 was designed to explore other factors likely to influence the frequency of nomination of exemplars.

\section{Study 3: Objective Attributes of Popular Exemplars}

Study 3 permitted a more detailed analysis of the five types of exemplars emerging from Study 1. Having judges evaluate all the exemplars would provide a more powerful design for understanding why certain exemplars were generated so often. Unfortunately, we found the knowledge base of the undergraduate students rather idiosyncratic: In a random sample of 22 undergraduate students from our subject pool, the mean recognition rate (yes/no) from a list of 50 famous exemplars of intelligence was only $42 \%$.

To avoid these complications, we sought the cooperation of judges knowledgeable enough to rate a diverse set of exemplars. Accordingly, in Study 3, two sets of knowledgeable judges were asked to rate the prototype and exemplar attributes of 50 of the exemplars collected from the local student sample in Study 1: The 50 were selected from the original 75 to maximize diversity while retaining the top 15. Regression analyses were then used to evaluate the independent contribution of each attribute in predicting previous exemplar frequencies.

Lay prototype variables. Previous research by Sternberg and colleagues has identified three prototypical features of lay prototypes of intelligence, namely, problem-solving ability, verbal ability, and social competence (Sternberg, 1985). Accordingly, we asked one set of judges to rate all 50 candidates on these three dimensions.

Exemplariness variables. To pursue the exemplar approach, we asked another set of knowledgeable judges to rate the degree to which each of the 50 candidates exemplified each of the five subgroups of intelligent exemplars. Rather than defining these rating dimensions with a label (e.g., scientific intelligence), we defined each dimension in terms of two exemplars from Study 1. A high degree of interjudge agreement would suggest that participants are making systematic and consensual use of exemplar information.

Finally, the nomination rate of these 50 exemplars (derived from Study 1 data) was regressed on these eight predictors (in addition to fame and gender) to evaluate the degree to which a candidate's nomination likelihood can be predicted from his or her (consensually defined) attributes.

\section{Method}

The fixed set of 50 exemplars was chosen to include the top 15 most common individuals over various samples in Study 1. The set included 35 others chosen for their diversity from the 77 remaining exemplars.

The predictor variables in the present study were aimed at relatively objective qualities of the exemplars qualities on which knowledgeable judges would agree. To bypass the limited and idiosyncratic knowledge base of our undergraduate student samples, we chose knowledgeable judges - mostly faculty and graduate students in psychology - on the basis of their interest and familiarity with these materials. The judges were told explicitly to ignore their personal preferences and provide ratings that captured the candidates' objective qualities. Moreover, they were instructed that if not sufficiently familiar with a candidate, they should take time to investigate relevant information.

All variables were rated on 7-point scales ranging from low (1) to high (7). One set of 10 judges rated fame, problem-solving ability, verbal intelligence, and social competence. Another set of 10 knowledgeable judges rated five kinds of exemplariness for each of the 50 candidates. The ratings of the judges were averaged for use as predictors in the regression analyses.

\section{Results and Discussion}

Exemplar frequencies. The frequencies (as percentages of total reports) were averaged over the three student samples in Study 1. Beforehand, a natural log transformation was used to normalize each of the distributions. The intraclass correlation of this mean was a strong .90 . This mean frequency (percentage of total) was then regressed on three blocks of predictors: (a) two control variables, (b) three lay prototype variables, and (c) five exemplariness variables. Note that the sample size is 50 because all correlations were calculated across the means for 50 exemplars (rather than across participants).

Control variables. The candidate's fame and gender were considered to be control variables. The intraclass correlation of the mean of the fame ratings was high (.85) and the reliability of gender is assumed to be 1.00. The two variables were entered as a block: They failed to make a significant contribution and therefore were dropped from consideration. ${ }^{4}$ Nonsignificance for nomi- 
Table 3. Regression of Nomination Likelihood on Three Lay Prototype Attributes in Study 3

\begin{tabular}{|c|c|c|c|c|c|c|}
\hline \multirow[b]{2}{*}{$\begin{array}{l}\text { Prototype } \\
\text { Attribute }\end{array}$} & \multirow{2}{*}{\multicolumn{2}{|c|}{$\begin{array}{c}\text { Regression } \\
\text { Coefficients } \\
\text { Beta }\end{array}$}} & \multirow[b]{2}{*}{$t$} & \multirow[b]{2}{*}{ Sig. } & \multicolumn{2}{|c|}{ Correlations } \\
\hline & & & & & Zero-Order & Partial \\
\hline \multicolumn{2}{|c|}{ Problem solving } & .29 & 2.38 & .02 & .37 & .33 \\
\hline \multicolumn{2}{|c|}{ Verbal ability } & .26 & 2.11 & .04 & .34 & .34 \\
\hline \multicolumn{2}{|c|}{ Social competence } & .31 & 2.50 & .02 & .40 & .35 \\
\hline
\end{tabular}

$N=50$ exemplars. All $t$ tests are one-tailed.

nee sex indicates that the mean exemplar rate of women on our list did not differ significantly from the mean of men on our list. Similarly, the fact that fame was not a significant predictor reflects the fact that a number of individuals receiving high fame ratings (e.g., Elvis, Pamela Anderson, Michael Jordan) were rarely nominated as exemplars of intelligence.

Prototype dimensions. The ratings of the first panel of knowledgeable judges were averaged to yield three lay prototype variables (problem-solving ability, verbal ability, social competence). All reliabilities were strong, as indicated by high intraclass correlations: problemsolving ability (.93), verbal intelligence (.89), and social competence (.86).

The three variables were entered simultaneously into an equation predicting exemplar frequency. Note from Table 3 that all three predictors reached significance at the .05 level. This result confirms that the 50 exemplars include individuals representing all three features of the lay prototype (Sternberg, 1988). Conversely, the result confirms that these three prototype features provide a meaningful way of distinguishing among exemplars.

Five types of exemplariness. Seven knowledgeable judges rated the 50 targets according to how well they represented of each of the five subtypes of intelligence, that is, scientific intelligence, artistic intelligence, entrepreneurial intelligence, communicative intelligence, and moral intelligence. The labels were not mentioned; instead, the types of intelligence were represented by two examples: Type A (Einstein, Hawking), Type B (Mozart, Shakespeare), Type C (Bill Gates, Donald Trump), Type D (Bill Clinton, Oprah), and Type E (M. L. King, Mother Theresa). We also asked these judges to describe the type of intelligence captured by each type A through E. Their labels largely substantiated our choices.

To index the degree of consensual meaning in these exemplars, we calculated correlations among the seven judges' ratings. The average interrater correlations were as follows: scientific (.89), artistic (.69), entrepreneurial (.63), communication (.75), and moral (.61). Such values
Table 4. Regression of Nomination Likelihood on Five Exemplariness Ratings in Study 3

\begin{tabular}{|c|c|c|c|c|c|}
\hline \multirow[b]{2}{*}{$\begin{array}{l}\text { Type of } \\
\text { Intelligence }\end{array}$} & \multirow{2}{*}{$\begin{array}{c}\text { Regression } \\
\text { Coefficients } \\
\text { Beta }\end{array}$} & \multirow{2}{*}{$t$} & \multirow[b]{2}{*}{ Sig. } & \multicolumn{2}{|c|}{ Correlations } \\
\hline & & & & Zero-Order & Partial \\
\hline Scientific & .39 & 3.64 & .001 & .48 & .48 \\
\hline Artistic & .38 & 3.41 & .001 & .27 & .45 \\
\hline Entrepreneurial & .31 & 2.36 & .01 & .21 & .33 \\
\hline Communicator & .21 & 1.68 & .05 & .27 & .24 \\
\hline Moral & .38 & 3.00 & .002 & .32 & .41 \\
\hline
\end{tabular}

$N=50$ exemplars. All $t$ tests are one-tailed.

indicate varying degrees of agreement among the judges in how much the targets represented each subtype of intelligence. Nonetheless, their composites all yielded alphas greater than .90 , thereby permitting averaging for further analysis.

These five mean exemplariness ratings were then used to predict the exemplar frequencies. The regression results are presented in Table 4. Note that all five exemplariness ratings emerged as significant predictors. The multiple correlation $(R=.64)$ is also very high, ${ }^{5}$ indicating that the five types of intelligence represent a near comprehensive taxonomy of famous exemplars.

\section{Study 4: Famous versus Nonfamous Exemplars of Intelligence}

Studies 1 and 2 required that exemplars be limited to famous individuals. Unknown is the degree to which other individuals (e.g., family members and friends) would have been reported as exemplars of intelligence. Such information is necessary to clarify the role of exemplars in everyday cognition. In Study 4 , therefore, we did not constrain nominees to be famous. We also collected various kinds of rating data to help interpret the choice of nonfamous exemplars.

\section{Method}

Exemplars of intelligence (one each) were collected from a total of 211 students at the University of British Columbia (UBC). The procedure was identical to that in Study 1 except that exemplars were not restricted to famous persons.

\section{Results and Discussion}

Exemplars were coded as famous if they had received some public recognition and therefore were potentially knowable to all our student reporters. They were coded as nonfamous only if the reporter had personally met them. The few public figures that had been met were excluded. An overall compilation of the relative proportions revealed $69 \%$ famous and $31 \%$ nonfamous exemplars. 


\section{Famous Exemplars}

To compare with Study 1, we compiled the famous exemplars separately. The same popular exemplars appeared near the top of the list (Einstein, Da Vinci, etc.). The similarity was confirmed by a strong correlation of these frequencies with the UBC exemplar report frequencies from Study $1(r=.93, p<.01)$. This strong similarity served to assure us (retroactively) that our decision to restrict nominations to famous persons in Study 1 did not affect the relative nomination rates of famous exemplars.

\section{Nonfamous Exemplars}

As noted, the nonfamous exemplars accounted for $31 \%$ of the total. Of those, the breakdown was as follows: acquaintances (22), teachers (20), close friends (16), fathers (15), mothers (9), brothers (9), sisters (5), and others (4). These relative proportions of family members nominated as intelligent are consistent with previous research on people's estimates of the IQs of their family members (Furnham, Fong, \& Martin, 1999). For example, fathers appear to be viewed as the most intelligent family member.

Multidimensional scaling. Parallel to Study 1, we asked a separate set of 16 undergraduate judges to rate the five individuals emerging from the nonfamous exemplar reports (smart acquaintance, smart teacher, good friend, father, mother, brother, sister, and one other intelligent acquaintance). Unfortunately, the correlations among the judges' similarity ratings were rather meager (mean $r=-.09$ ), thereby precluding a scaling of this sort. Of course, this lack of convergence is not surprising given that perceptions of family members and friends are inherently idiosyncratic. Although Einstein may represent a similar form of intelligence to most judges, one's best friend or brother does not. Future analyses of this sort will require simultaneous ratings of famous and nonfamous exemplars by the same judges. Because the famous exemplars yield a more robust structure across individuals, they could be used to interpret the meaning of the nonfamous exemplars. These analyses await further data.

Exemplariness of nonfamous exemplars. Instead, we polled another 66 undergraduate judges using the exemplariness technique described earlier. Each judge was asked to consider the five types of intelligence A through E personified by exemplar pairs: Einstein/ Hawking, Mozart/Shakespeare, Gates/Trump, Clinton/Winfrey, and King/Mother Theresa. All confirmed that they recognized the exemplars with the exception that $15 \%$ did not recognize Stephen Hawking and 3\% did not recognize Trump or M. L. King. ${ }^{6}$
They were asked to rate the intelligence of six nonfamous individuals (a good friend, mother, father, oldest brother or sister, an acquaintance, and a teacher) in terms of the five kinds of intelligence. The instructions were similar to those in Study 1. Results showed that teachers were ranked highest on scientific and communicator intelligence, fathers on entrepreneurial intelligence, mothers on moral intelligence, and friends on artistic intelligence.

These analyses were merely exploratory: Any incisive analysis of significant others will require the addition of other measures along with exemplar reports and ratings. For example, the idiosyncratic nature of personal spaces can be dealt with by collecting and analyzing an individual's personal constructs (Kelly, 1963). Among the intriguing possibilities is that, compared to nonacquaintances, exemplars who are personal acquaintances will have a stronger impact on aspirations and self-definition. Another possibility is that attachment styles bear a systematic relation to choice of family members as exemplars of the five types of intelligence.

\section{General Discussion}

Along with Robert Sternberg (e.g., 1988), Howard Gardner has led the recent movement to broaden traditional conceptions of intelligence (e.g., Gardner, 1993, 1998). To communicate his arguments for nine distinct types of intelligence, Gardner has made effective use of famous exemplars such as Einstein, Beethoven, Freud, and Darwin. ${ }^{7}$ A reading of his work reveals the tight interplay of conceptions and exemplars in developing his ideas. Accordingly, Gardner's nine types provide a valuable template for comparison with our types.

Although Gardner's approach was more rational than empirical, his claims concerned real-world differences in types of intelligence. Our work was explicitly inductive and empirical but our claims concern implicit types of intelligence. We used the exemplar survey method (Paulhus \& Landolt, 2000) and collected large surveys of popular exemplars. As detailed below, our four studies supported some of Gardner's conclusions but not others. ${ }^{8}$

\section{Consistency Across Samples}

One notable finding from Paulhus and Landolt (2000) was a substantial consistency across 16 years in the most popular exemplars of intelligence. An alternative explanation for the observed temporal stability is that all surveys were conducted at the same school in the same country. In the present studies, therefore, we began by comparing samples from a variety of North American colleges. We also added a relatively educated adult sample. Among the most frequently reported exemplars, the differences across time and samples were minor. A com- 
mon group of nominees dominated all four lists. Not only are nomination rates temporally stable but they also converge sufficiently to indicate substantial consensus on the implicit definition of intelligence among educated North Americans.

\section{Distinctiveness From Other Constructs}

In Study 2, we examined the popular exemplars of intelligence for evidence of discriminant validity with respect to other appealing qualities of the nominees, namely, their fame, creativity, and wisdom. When we compared a sample of nominees for intelligent person with a sample of nominees for a famous person, only a $30 \%$ overlap was observed in top 15 lists. A comparison of popular exemplars for Sternberg's (1988) two other forms of mental ability revealed minor, although systematic, overlap across the popular exemplars of intelligence, creativity, and wisdom. These findings are critical to verify that judges are not indiscriminately nominating popular and/or famous people.

The fact that creativity showed the largest overlap with intelligence is consistent with our Study 1 finding that artistic intelligence emerged as one of the five types of intelligence. Moreover, our overall pattern of overlap matched previous estimates of overlap using the prototype approach (Sternberg, 1985). In sum, the same pattern of distinctiveness previously demonstrated in judges' conceptions of intelligence, creativity, and wisdom was confirmed here in our judges' selections of ideal exemplars of those concepts.

Together, these preliminary studies of exemplars of intelligence support arguments that members of a common culture - in this case North Americans with at least some degree of college education - have similar conceptions of intelligence (see Berry, 1984; Sternberg, 1988, p. 46). When exemplars did lose their popularity over time, they were replaced with an exemplar of the same type. Lee Iacocca, for example, was replaced by Donald Trump and later by Bill Gates (Paulhus \& Landolt, 2000). This stability in exemplars of intelligence represents a "shared cognition" that serves to facilitate communication, social interactions, and group decisions (see Romney \& Moore, 1998; Thompson \& Fine, 1999).

Such implications are weakened by the fact that nominations in Studies 1 and 2 were restricted to famous people. In Study 4, that restriction was lifted with the result that $31 \%$ of the exemplars were nonfamous, that is, friends, acquaintances, family members, teachers, and so forth, and the patterns were coherent; for example, fathers best represented scientific intelligence and mothers best represented moral intelligence.

Our results do not reveal the relative ranks or roles that individuals give to famous and nonfamous exemplars, and we do not know what kinds of individuals nominate each. Note that we have used the term famous to mean "public" - not necessarily universally known. In fact, as noted earlier, the average undergraduate recognized only $42 \%$ of the famous exemplar list. From this perspective, the apparent consensus in famous exemplars is due, at least in part, to a limited common knowledge base, even among the educated. We suspect that the more intellectually sophisticated among our judges tended to report the more esoteric exemplars.

\section{The Structure of Diversity}

Despite their consistency across time and subgroups, the diversity of the intellectual paragons was striking: Individuals as varied as Einstein, Shakespeare, Oprah Winfrey, Bill Gates, Madonna, Bill Clinton, and Mahatma Gandhi received large numbers of nominations. These individuals may well represent distinct subcultures in contemporary society. In any case, no one field holds a monopoly on icons of intelligence.

To clarify this stable diversity of exemplars, we conducted a multidimensional scaling of judges' similarity ratings. The underlying perceptual dimensions were not easy to interpret. Instead, the striking feature of these analyses was the clustering of five subgroups of exemplars. Based on the exemplars, the implicit types of intelligence were labeled as scientific, artistic, communicative, entrepreneurial, and moral intelligences.

Based on the similarity of content and exemplars, several of our implicit types can reasonably be mapped onto Gardner's nine intelligences. Our scientific intelligence resembles his logical-mathematical (Einstein), our artistic intelligence subsumes his musical and linguistic (Shakespeare and Mozart), our communicative intelligence resembles his interpersonal (presidents), and our moral intelligence resembles his existential intelligence (Dalai Lama). We found little support in the judgments of our students for the distinctiveness of Gardner's spatial, kinesthetic, intrapersonal, or naturalist types of intelligence. In addition, unlike our lay judges, Gardner has never proposed entrepreneurial acumen as a distinct form of intelligence.

In Study 3, we showed that judges were able to use exemplars of each of the five types of intelligence to guide their judgments of other famous individuals. In Study 4, lay judges used the exemplars to guide judgments of friends, family, and teachers. The meaningfulness of these judgments was confirmed by the high interjudge agreement and the ability of the composite ratings to predict actual exemplar frequencies.

\section{Fame, Salience, and Nomination Rates}

Are the most commonly reported exemplars simply high profile icons of contemporary society? Several pieces of evidence suggest not. First, direct nomi- 
nations of famous individuals in Study 2 yielded a list dominated by such cultural icons as Elvis, Ali, and Princess Diana - individuals rarely nominated as exemplars of intelligence. Study 2 also was informative in showing relatively distinct lists of nominees for intelligence, creativity, and wisdom: Such distinctiveness could not have resulted from use of a single salience heuristic to make nominations. Finally, in Study 3, fame was tested but failed to predict exemplar rates. In sum, not all creative, wise, or famous people make good exemplars of intelligence.

We have no doubt that popular exemplars tend to be famous people. Highly available individuals are more likely to be used as exemplars. Thus, availability is a necessary but not sufficient condition for popularity as an exemplar of intelligence. In addition, the exemplar does not have to be available to society as a whole, just available to nominators. Each judge's exemplars are prominent in his or her own world. Thus, Paulhus and Landolt (2000) found that choice of exemplars was highly correlated with rated fame when calculated within individuals. The putative requirement of public fame is refuted directly by the third of our sample who chose family members and friends as their exemplars of intelligence.

Conversely, there are many icons of undeniable genius (Spinoza, J. S. Mill, M. Curie, etc.) whose current fame was insufficient to draw many nominations. Rather than a deficiency of the current methodology, this absence is simply a demonstration that absolute ability and exemplar popularity are not isomorphic. Obscurity is a strong impediment to the nomination of and (we would argue) the use of exemplars.

\section{Implications for Cognition}

We are left with some tentative implications about implicit theories of intelligence. As with other important concepts, internal representations of the trait "intelligent" appear to include specific exemplars. Those exemplars are activated independently of exemplars of other mental abilities. An individual's exemplars must be salient to the perceiver but not necessarily famous across the culture. They need not even be public figures - family and friends are a major source of exemplars.

Our expert judges in Study 1 were able to distinguish five subtypes of intelligence: scientific, artistic, entrepreneurial, communicative, and moral, and in Study 4, another set of judges found these conceptions sufficiently meaningful to provide relatively consensual but orthogonal ratings. Moreover, a resemblance to any of the five types predicted high nomination ratings. Note that such analyses are possible only within an exemplar approach to target assessment (Broughton, 1984).

Our five types of implicit intelligence do not map easily onto the three lay prototype features of intelligence, namely, problem solving, verbal ability, and social competence (Sternberg, 1988). Instead, our intelligence types involve combinations of that trio of abilities. For example, the charismatic leadership embodied by communicative intelligence would require both verbal ability and social competence. Entrepreneurial intelligence might weight problem solving heavily but could well include verbal ability and social competence.

Surprising to some readers, no doubt, is the emergence of a distinct moral intelligence. Nonetheless, in the eyes of (many of) our relatively educated judges, individuals such as Martin Luther King, Mahatma Gandhi, Mother Theresa, Jesus Christ, the Dalai Lama, and the Pope exemplify intelligence. This finding resonates with recent arguments by Emmons (2000) that the ability to grasp spiritual ideas and apply spiritual resources to everyday problems is rightfully considered a form of intelligence. Such a finding is a reminder that lay observers do not share the traditional psychometric tendency to segregate personality from intelligence: They see much overlap between the two domains (Ackerman \& Heggestad, 1997; Paulhus et al., 1998).

Our exemplar approach is not inconsistent with the more established prototype-feature approaches to conceptions of traits (e.g., Broughton, 1984; Chaplin et al., 1988; Fehr, 1988; Sternberg, 1985). In fact, with available techniques, it has been difficult to establish whether cognitive representations of traits are primarily feature based (prototype-like), exemplar based, or some combination of the two (Barsalou, 1990; Smith \& Zarate, 1992). For many purposes, of course, it is immaterial whether features precede exemplars, or vice versa, because once raised, an exemplar feeds back onto the storage of features (Smith \& Zarate, 1990). Nonetheless, one direction for future research would be to study the cognitive consequences of inducing participants to process specific exemplars.

Future research will also have to clarify the relation between the overt report of an exemplar and the underlying representation. One could accept reported exemplars at face value; that is, whatever process underlies a report, the final product constitutes an accurate indicator of the exemplar content of the nominator's implicit theory of intelligence. Ultimately, however, future research will have to tease apart the roles played by such mechanisms as value expression (Buss \& Briggs, 1984), person memory (Mayer \& Bower, 1986), impression management (Paulhus, 1991), narcissistic bias (Robins \&John, 1997), and automaticity (Logan, 1988). An overt response to an exemplar request may well hide a multitude of whims.

\section{Future Directions}

We have initiated an empirical exploration of lay exemplars of intelligence - a topic given little previous at- 
tention. Our earlier work (Paulhus, 2000; Paulhus \& Landolt, 2000) provided initial evidence that famous exemplars might play a role in lay theories of intelligence. The present report expanded on those ideas by demonstrating (a) a consistency across samples and (b) a structured diversity within those popular paragons. The results of our research suggest that exemplars do play a role in cognitive representations of intelligence. They suggest further that exemplar surveys can reveal implicit types. A similar methodology could be applied to other trait representations.

Some of our exemplar results complement and support previous work using a prototype approach. Some of the results go well beyond the earlier work. The finding that implicit theories can entertain five distinct types of intelligence is a novel finding. The emergence of moral intelligence as a distinct form is most striking. So too is the absence of evidence for spatial, kinesthetic, naturalist, and intrapersonal intelligences as distinct forms. Finally, we see no simple mapping of any of our five types onto the currently popular notion of emotional intelligence.

Further work is required to determine whether the five subtypes of intelligence derive from (a) five subcultures of judges each valuing one form of intelligence or (b) five categories operating in any judge. Within-person clustering would address whether implicit types exist at the individual as well as the group level. Most likely is that some judges can entertain multiple subtypes of intelligence, whereas others can entertain only one type (Tetlock \& Suedfeld, 1988).

Although we have identified the implicit types, more fundamental laboratory work is required to elucidate the role of exemplars in implicit theories of intelligence. Ideally, exemplar and prototype information should be manipulated independently to examine effects of judgments of intelligence (and more generally, the processes involved in making intelligence judgments). Are judgments of intelligence facilitated after generating exemplars and/or after generating prototypes of intelligence? Similarly, does priming a particular exemplar category affect intelligence ratings? For example, if scientific intelligence is primed, are Einstein and Hawking rated more quickly and are they perceived as more intelligent than if another category is primed? Does irrelevant exemplar information (e.g., Einstein's German accent and moustache) eventually become imbued with intelligence?

We encourage others to examine possible links between implicit types and previously established aspects of implicit theories of intelligence. One is the influential distinction between entity and incremental lay theories (e.g., Dweck, 1996). Do lay entity and incremental theorists use different exemplars - ones exemplifying innate genius versus hard work, respectively? Another potential connection is with defensive self-definitions of intel- ligence demonstrated in the research by Dunning and colleagues (e.g., Dunning, Meyerowitz, \& Holzberg, 1989). When individuals are threatened by a current definition of intelligence, ${ }^{9}$ do their exemplars switch along with the definitional attributes?

\section{Broader Implications}

At the cultural level, the present research has implications for the sociology of intellectual icons. Recall C. P. Snow's (1959) influential distinction between two distinctive forms of intelligence-scientific and literary. He argued that these two aspects of Western culture represented two groups and modes of thought that were almost mutually incomprehensible to one another. Our work suggests an even more complex set of five views of intelligence operating simultaneously in our sample of educated observers. ${ }^{10}$ Ultimately, they represent five prominent values, each generating a conception of intelligence that advances the value. Although they are necessarily determined by social history (Simonton, 1994; Snow, 1959), popular exemplars may, in turn, feed back onto social events (Gardner, 1993; Ruzgis \& Grigorenko, 1994). By association, currently popular exemplars may indirectly influence the image of various foreign countries, their citizens, their dress, and their accents, and the centrality of their exemplars in language and culture may modulate any trend toward a change in values. ${ }^{11}$ Once the name "Einstein" rather than Shakespeare came to personify intelligence in the Western world, the relative impact of science and literature in the 20th century may have been largely determined.

Finally, our demonstration of diversity within stable clusters of intellectual paragons recapitulates the current tension between diversity and standardization in intellectual circles. Diversity advocates warn of the restrictive effects of teaching only traditional icons. Standardization partisans argue that common icons provide a common dialect for discussions of intellectual achievement (Bloom, 1988). In this spirit, Hirsch (1988) has argued that, arbitrary or not, such icons provide the linguistic currency necessary for a flourishing culture. Our demonstration of a limited number of worldviews suggests that a judicious selection of five intellectual icons would suffice.

\section{Notes}

1. Other advances in implicit theories of intelligence include determining its structural location in implicit theories of personality (Rosenberg \& Sedlak, 1972), the self-serving nature of its definitions (Dunning, Meyerowitz, \& Holzberg, 1989), and the entity-incremental distinction (e.g., Dweck, 1996).

2. To make the 15-person list, nominees had to rank in the top 25 on several surveys. 
3. Although not in the top 15, Einstein was close on the wisdom list and King was close on the intelligence list. Indeed, King did make the overall top 15 in Study 1.

4. Nonsignificant control variables should not be retained in subsequent steps. Otherwise, a valid portion of variance may be discarded.

5. Note that the maximum attainable value is only .77 because neither the criterion nor the predictors are perfectly reliable.

6. In all these cases, judges recognized the other paired exemplar allowing them to do the ratings.

7. His nine intelligences with exemplars were linguistic (T. S. Eliot, Chomsky, Auden, Angelou), logical-mathematical (Einstein, Whitehead, Curie, Ulam, Poincaré), musical (Stravinsky, Beethoven, Bernstein, Midori, Coltrane), spatial (Michelangelo, F. L. Wright, Kasparov, H. Frankenthaler, H. Nevelson), kinesthetic (M. Graham, M. Jordan, M. Marceau), interpersonal (Ghandi, Eleanor Roosevelt, Mao), personal (Freud, Mead, Proust), naturalist (Darwin), and existential (Dalai Lama, Sartre, Kierkegaard).

8. Note that our data collection began before the appearance of Gardner's work.

9. This near-universal tendency to label oneself as intelligent helps explain the rather modest correlation between self-perceptions and IQ tests (Paulhus, Lysy, \& Yik, 1998).

10. Note again that Gardner's is an explicit theory of intelligence: It claims that multiple types of intelligence actually exist. Our work addresses variations in implicit theories, that is, lay conceptions of intelligence.

11. Note that there is no need to validate the actual intellectual ability of exemplars. The only issue is whether society attributes intelligence to them (Kasof, 1995).

\section{References}

Ackerman, P. L., \& Heggestad, E. D. (1997). Intelligence, personality, and interests: Evidence for overlapping traits. Psychological Bulletin, 121, 219-245.

Banaji, M. R., \& Greenwald, A. G. (1995). Implicit gender stereotyping in judgments of fame. Journal of Personality and Social Psychology, 68, 181-198.

Barsalou, L. W. (1990). On the indistinguishibility of exemplar memory and abstraction in category representation. In T. K. Srull \& R. S. Wyer (Eds.), Advances in social cognition (Vol. 3). Hillsdale, NJ: Lawrence Erlbaum.

Berry, J. W. (1984). Towards a universal psychology of cognitive competence. International Journal of Psychology, 19, 335-361.

Bloom, A. (1988). The closing of the American mind. New York: Simon \& Schuster.

Broughton, R. (1984). A prototype strategy for construction of personality scales. Journal of Personality and Social Psychology, 47, 1334- 1346.

Buss, A. H., \& Briggs, S. R. (1984). Drama and the self in social interaction. Journal of Personality and Social Psychology, 47, 1310-1324.

Cantor, N., \& Mischel, W. (1979). Prototypes in person perception. In L. Berkowitz (Ed.), Advances in experimental social psychology (Vol. 12, pp. 3-52). New York: Academic Press.

Chaplin, W. F., John, O. P., \& Goldberg, L. R. (1988). Conceptions of traits and states: Dimensional attributes with ideals as prototypes. Journal of Personality and Social Psychology, 54, 541-557.
Dunning, D., Meyerowitz, J. A., \& Holzberg, A. D. (1989). Ambiguity and self-evaluation: The role of idiosyncratic trait definitions in self-serving assessments of ability. Journal of Personality and Social Psychology, 61, 1082-1090.

Dweck, C. S. (1996). Implicit theories as organizers of goals and behavior. In P. M. Gollwitzer \& J. M. Bargh (Ed.), The psychology of action: Linking cognition and motivation to behavior (pp. 69-90). New York: Guilford.

Emmons, R. A. (2000). Is spirituality an intelligence? Motivation, cognition, and the psychology of ultimate concern. International Journal for the Psychology of Religion, 10, 3-26.

Fehr, B. A. (1988). Prototype analysis of the concepts of love and commitment. Journal of Personality and Social Psychology, 55, 557-579.

Furnham, A., Fong, G., \& Martin, N. (1999). Sex and cross-cultural differences in the estimated multifaceted intelligence quotient score for self, parents and siblings. Personality and Individual Differences, 26, 1025-1034.

Gardner, H. (1993). Creating minds: An anatomy of creativity seen through the lives of Freud, Einstein, Picasso, Stravinsky, Eliot, Graham, and Gandhi. New York: Basic Books.

Gardner, H. (1998). A multiplicity of intelligences. Scientific American, 9, 19-23.

Hirsch, E. D. (1988). Cultural literacy. New York: Vintage.

Hogan, R., \& Hogan, J. (1994). Personality and status. In D. G. Gilbert \& J. J. Connolly (Eds.), Personality, social skills and psychopathology: An individual differences approach. New York: Plenum.

Holliday, S. G., \& Chandler, M. J. (1986). Wisdom: Explorations in adult competence. Contributions to Human Development, 17, 100- 113.

Hong, Y. Y., Chiu, C. Y., \& Dweck, C. S. (1995). Implicit theories of intelligence: Reconsidering the role of confidence in achievement motivation. In M. H. Kernis (Ed), Efficacy, agency, and self-esteem (pp. 197-216). New York: Plenum.

Jacoby, L. L., Kelley, C., Brown, J., \& Jasechko, J. (1989). Becoming famous overnight: Limits on the ability to avoid unconscious influences of the past. Journal of Personality and Social Psychology, 56, 326- 338.

John, O. P. (1986). How shall a trait be called: A feature analysis of altruism. In A. Angleitner, A. Furnham, \& G. Van Heck (Eds.), Personality psychology in Europe: Current trends and controversies (pp. 117-140). Berwyn, PA: Swets North America.

Kasof, J. (1995). Explaining creativity: The attributional perspective. Creativity Research Journal, 8, 311-366.

Kelly, G. A. (1963). The psychology of personal constructs. New York: Norton.

Kosmitzki, C., \& John, O. P. (1993). The implicit use of explicit conceptions of social intelligence. Personality and Individual Differences, 15, 11-23

Logan, G. D. (1988). Toward an instance theory of automatization. Psychological Review, 95, 492-597.

Mayer, J. D., \& Bower, G. H. (1986). Learning and memory for personality prototypes. Journal of Personality and Social Psychology, 51, 473- 492.

Neisser, U. (1979). The concept of intelligence. In R. J. Sternberg \& D. K. Detterman (Eds.), Human intelligence: Perspectives on its theory and measurement (pp. 179-189). Norwood, NJ: Ablex. 
Nosofsky, R. M. (1992). Exemplar-based approach to relating categorization, identification, and recognition. In F. G. Ashby (Ed.), Multidimensional models of perception and cognition (pp. 363-393). Hillsdale, NJ: Lawrence Erlbaum.

Paulhus, D. L. (1991). Measurement and control of response bias. In J. P. Robinson, P. Shaver, \& L. S. Wrightsman (Eds.), Measures of personality and social psychological attitudes (pp. 17-59). San Diego, CA: Academic Press.

Paulhus, D. L. (2000). Nominations of intelligent exemplars: Contributions of target achievement and prototype fit. Social Cognition, 18, 319-328.

Paulhus, D. L., \& Landolt, M. A. (2000). Paragons of intelligence: Who gets nominated and why. Canadian Journal of Behavioural Science, 32, 168-177.

Paulhus, D. L., Lysy, D. C., \& Yik, M. S. M. (1998). Self-report measures of intelligence: Are they useful as proxy IQ tests? Journal of Personality, 66, 525-554.

Raty, H., \& Snellman, L. (1997). Children's images of an intelligent person. Journal of Social Behavior and Personality, 12, 773-784.

Robins, R. W., \& John, O. P. (1997). The quest for self-insight: Theory and research on accuracy and bias in self-perception. In R. Hogan, J. Johnson, \& S. R. Briggs (Eds.), Handbook of personality psychology (pp. 649-679). San Diego, CA: Academic Press.

Romney, A. K., \& Moore, C. C. (1998). Toward a theory of culture as shared cognitive structures. Ethos, 26, 314-337.

Rosenberg, S., \& Sedlak, A. (1972). Structural representations of perceived personality trait relationships. In A. K. Romney, R. N.

Shepard, \& S. B. Nerlove (Eds.), Multidimensional scaling (pp. 134- 162). New York: Seminar Press.

Ruisel, I. (1996). Implicit theories of intelligence in adolescents. Studia Psychologica, 38, 23-34.
Rush, M. C., \& Russell, J. E. A. (1988). Leader prototypes and prototype- contingent consensus in leader behavior descriptions. Journal of Experimental Social Psychology, 24, 88-104.

Ruzgis, P., \& Grigorenko, E. L. (1994). Cultural meaning systems, intelligence, and personality. In R. J. Sternberg \& P. Ruzgis (Eds.), Personality and intelligence (pp. 248-270). New York: Cambridge University Press.

Simonton, D. K. (1994). Greatness: Who makes history and why. New York: Guilford.

Smith, E. R., \& Zarate, M. A. (1990). Exemplar and prototype use in social categorization. Social Cognition, 8, 243-262.

Smith, E. R., \& Zarate, M. A. (1992). Exemplar-based model of social judgment. Psychological Review, 99, 3-21.

Snow, C. P. (1959). The two cultures and the scientific revolution. New York: New American Library.

Sternberg, R. J. (1985). Implicit theories of intelligence, creativity, and wisdom. Journal of Personality and Social Psychology, 49, 607-627.

Sternberg, R. J. (1988). The triarchic mind: A new theory of human intelligence. New York: Penguin Books.

Sternberg, R. J., Conway, B. E., Ketron, J. L., \& Bernstein, M. (1981). People's conceptions of intelligence. Journal of Personality and Social Psychology, 41, 37-55.

Tetlock, P. E., \& Suedfeld, P. (1988). Integrative complexity coding of verbal behaviour. In P. E. Tetlock, P. Suedfeld, \& C. Antaki (Eds.), Analysing everyday explanation: A casebook of methods (pp. 43-59). London: Sage Ltd.

Thompson, L., \& Fine, G. A. (1999). Socially shared cognition, affect, and behavior: A review and integration. Personality and Social Psychology Review, 3, 278-302.

Tversky, A., \& Kahneman, D. (1974). Judgment under uncertainty: Heuristics and biases. Science, 185, 1124-1131. 